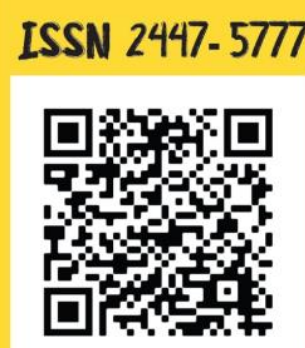

$\varepsilon$

M

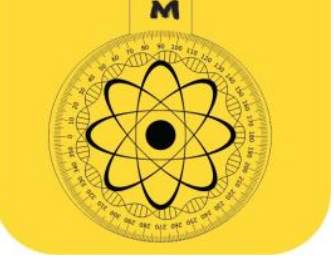

ENSINO \&

\title{
MULTIDISCIPLINARIDADE
}

Jan. | Jun. 2021, Volume 7, Número 1, p. 111-130.

\section{Modelo teórico de aproximações para o ensino de Ciências entre as premissas da História da Ciência e do pensamento crítico}

\author{
Theoretical model of approaches for the teaching of Science between the \\ premisses of the History of Science and critical thinking
}

\author{
Sigouveny Cruz Cardoso ${ }^{1}$ - https://orcid.org/0000-0001-5879-7081 \\ Erivanildo Lopes da Silva ${ }^{2}$ - https://orcid.org/0000-0003-2207-8661
}

${ }^{1}$ Mestre em Ensino de Ciências e Matemática pela Universidade Federal de Sergipe (PPGECIMA/UFS).

Professora Colaboradora Voluntária do Departamento de Química da Universidade Federal de Sergipe

(DQI/UFS), São Cristóvão, Sergipe, Brasil. E-mail: sigouveny15@gmail.com

${ }^{2}$ Doutor em Ensino, Filosofia e História das Ciências pela Universidade Federal da Bahia (PPGEFHC/UFBA).

Professor Adjunto do Departamento de Química da Universidade Federal de Sergipe (DQI/UFS), São Cristóvão,

Sergipe, Brasil. E-mail: erivanildo@academico.ufs.br

\begin{abstract}
Resumo
No ensino de Ciências, o conhecimento científico para a tomada de decisão pode ser um fator preponderante para fundamentar o desenvolvimento de abordagens metodológicas para a resolução de problemas em sala de aula. Desse modo, a História da Ciência é considerada uma abordagem fundamental para essa contextualização do conhecimento científico em propostas didáticas que visem o desenvolvimento de habilidades cognitivas relacionadas ao pensamento crítico dos estudantes. Neste estudo, considera-se complexa a elaboração de propostas didáticas para o ensino de Ciências sem um modelo que direcione as atividades para tal finalidade. Então, esta pesquisa objetiva estabelecer aproximações teóricas entre a História da Ciência e o pensamento crítico para o ensino de Ciências, propondo um modelo teórico com articulações entre seus objetivos. Ao considerar o conhecimento para a tomada de decisão como um aspecto central dessas aproximações, o estudo apresenta correlações implícitas e explícitas para o planejamento de propostas didáticas. Essas articulações teóricas possuem implicações consideradas fundamentais para a educação científica, em virtude de apresentar um modelo teórico para ensinar Ciências pelas dimensões teóricas de análise, problematização e distinção de informações científicas, ao materializá-las em atividades de aprendizagem que viabilizem a tomada de decisão e a resolução de problemas científicos.
\end{abstract}

Palavras-chave: História da Ciência. Pensamento Crítico. Aproximações teóricas. Ensino de Ciências.

Como citar: CARDOSO, S. C.; SILVA, E. L. A. Modelo teórico de aproximações para o ensino de Ciências entre as premissas da História da Ciência e do pensamento crítico. Ensino \& Multidisciplinaridade. v. 7, n. 1, p. 111-130, 2021. 


\begin{abstract}
In Science education, scientific knowledge for decision-making can be a major factor to support the development of methodological approaches to problem solving in the classroom. In this way, the History of Science is considered a fundamental approach for this contextualization of scientific knowledge, in didactic proposals that aim at the development of cognitive skills related to students' critical thinking. In this study, the elaboration of didactic proposals for the teaching of Science is considered complex, without a model that directs activities for this purpose. So, this research aims to establish theoretical approaches between the History of Science and critical thinking, for the teaching of Science, proposing a theoretical model with articulations between its objectives. When considering knowledge for decision making as a central aspect of these approaches, the study presents implicit and explicit correlations for the planning of didactic proposals. These theoretical articulations have implications considered fundamental for scientific education, as they present a theoretical model for teaching Science through the theoretical dimensions of analysis, problematization and distinction of contextualized scientific information, when materializing them in learning activities that enable decision-making and solving scientific problems.
\end{abstract}

Keywords: History of science. Critical thinking. Theoretical approaches. Science teaching.

\title{
Introdução
}

Ao considerar as premissas e expectativas de uma formação para a cidadania, a educação científica pode ser compreendida como um processo no qual as propostas de ensino refletem uma perspectiva futura, que pode estar distante da realidade contemporânea que cerca os problemas do processo de ensino e aprendizagem de Ciências, além de ocasionar diversas interpretações sobre seus objetivos para a formação científica devido à multiplicidade de sentidos atribuídos ao termo cidadania (CACHAPUZ et al., 2005; MOURA; GUERRA, 2016).

Por esses pressupostos, pesquisas no ensino de Ciências ressaltam que o desenvolvimento de estudantes críticos e participativos na sociedade pode estar atrelada à formação para a cidadania, de modo a considerá-la como uma meta a ser atingida para uma educação científica de qualidade (CACHAPUZ et al., 2005; MARTINS, 2006; SILVA, 2014; TENREIRO-VIEIRA; VIEIRA, 2000, 2005, 2021).

Isso significa que o ensino de Ciências requer abordagens fundamentadas em proposições teóricas que estimulem os estudantes a participarem das atividades, e que essas propostas precisam envolver a aquisição de conhecimentos científicos e o desenvolvimento de habilidades cognitivas de Pensamento Crítico (PC) para tornar possível a significação, a construção e a aplicação de conhecimentos científicos em ações conscientes de resolução de problemas e tomada de decisão (CACHAPUZ et al., 2005; MATTHEWS, 1995; TENREIROVIEIRA; VIEIRA, 2000).

Em face da dinamicidade dos sistemas que regem a sociedade, e considerando que isso se projeta no âmbito da sala de aula, é preciso refletir e questionar sobre "o quê" e "como" fazer para materializar, em propostas de ensino, o conhecimento científico e as habilidades cognitivas que são necessários para o desenvolvimento de uma formação crítica dos estudantes em sala de aula (FREIRE, 2007; HALPERN, 1999; TENREIRO-VIEIRA; VIEIRA, 2000).

Este estudo procura enfatizar a importância de que essas abordagens sejam realizadas de forma integrada no ensino de Ciências, pois, quando são consideradas de forma dissociada, podem constituir obstáculos para a compreensão da ciência e do funcionamento da atividade científica pelos estudantes, e estes precisam ter uma formação científica suficiente para desenvolver habilidades e para lidar com essas informações de forma consciente e autônoma (CACHAPUZ et al., 2005; LIPMAN, 1988; TENREIRO-VIEIRA; VIEIRA, 2000, 2005).

Nesse sentido, a aquisição do conhecimento científico e o desenvolvimento de 
habilidades cognitivas para a resolução de problemas podem ser considerados em abordagens baseadas em um corpo de conhecimentos teóricos articulados, que objetivem de forma explícita o ensino de Ciências, a exemplo da abordagem de História da Ciência (HC), para que a partir desses conhecimentos sejam projetadas as atividades de aprendizagem orientadas para o desenvolvimento de habilidades cognitivas, pela mobilização do Pensamento Crítico (CARDOSO, 2021; MATTHEWS, 1995; TENREIRO-VIEIRA; VIEIRA, 2019).

Apesar de ser considerado como um objetivo fundamental para a educação científica, essa abordagem orientada requer a explanação dos objetivos em comum de História da Ciência e do pensamento crítico antes da produção de materiais didáticos, pois essa base teórica pode delimitar os aspectos de sua proposição no ensino de Ciências (CARDOSO, 2021; LIPMAN, 1988; MATTHEWS, 1994; TENREIRO-VIEIRA; VIEIRA, 2000, 2005).

Desse modo, neste estudo procura-se responder a esta questão de pesquisa: "Dos referenciais selecionados, quais dos seus objetivos para o ensino de Ciências que podem possibilitar uma aproximação teórica de História da Ciência e do pensamento crítico?" Conforme os propósitos de Matthews $(1994,1995)$ e Tenreiro-Vieira e Vieira $(2019,2021)$, essa articulação incide nos aspectos teóricos da História da Ciência para a abordagem do conhecimento científico, de forma orientada para o desenvolvimento de habilidades de pensamento crítico no ensino de Ciências.

\section{A História da Ciência para a abordagem do conhecimento científico}

Apesar de ser bastante demarcada e difundida por pesquisas na área do ensino de Ciências, a História da Ciência apresenta uma diversidade de abordagens, sendo recorrente que tais abordagens privilegiem a ciência como uma atividade que em sua essência é desenvolvida por seres humanos. Assim, também é preciso discutir quais seriam as possibilidades didáticas que essa afirmação pode implicar nas propostas de ensino (GURGEL, 2020; MARTINS, 2006; MATTHEWS, 1994, 1995, 2002).

No ensino de Ciências, a História da Ciência tem sido apontada como um viés para humanizar a ciência e aproximá-la de outras atividades desenvolvidas no contexto social, essa afirmação poderia significar que a sua abordagem seria uma tarefa simples de ser realizada por professores no âmbito de sua prática educativa, porém, essa tarefa está longe de ser simples, e pode ser acompanhada por uma complexa empreitada nas proposições didáticas para o ensino de Ciências (MARTINS, 2007; MATTHEWS, 1994, 1995). Nesse sentido, Matthews tem sido a base para afirmar que:

\footnotetext{
A história, a filosofia e a sociologia da ciência não têm todas as respostas para essa crise, porém possuem algumas delas: podem humanizar as ciências e aproximá-las dos interesses pessoais, éticos, culturais e políticos da comunidade; podem tornar as aulas de ciências mais desafiadoras e reflexivas, permitindo, deste modo, o desenvolvimento do pensamento crítico; podem contribuir para um entendimento mais integral de matéria científica, isto é, podem contribuir para a superação do mar de falta de significação que se diz ter inundado as salas de aula de ciências, onde fórmulas e equações são recitadas sem que muitos cheguem a saber o que significam; podem melhorar a formação do professor auxiliando o desenvolvimento de uma epistemologia da ciência mais rica e mais autêntica, ou seja, de uma maior compreensão da estrutura das ciências bem como do espaço que ocupam no sistema intelectual das coisas (1995, p. 165).
}

Por essa perspectiva, a abordagem da História da Ciência no ensino de Ciências tende a superar visões anedóticas e distorcidas de grandes heróis de nomes da ciência, ou até mesmo 
aquelas que relatam eventos históricos com base em conhecimentos científicos aceitos atualmente (ALLCHIN, 2004; MARTINS, 2006; MATTHEWS, 1995).

No ensino de Ciências, essa visão distorcida não considera uma abordagem dos embates entre filósofos ou cientistas de um determinado período, as discussões com a comunidade científica, as diferentes hipóteses e teorias elaboradas para estudar um determinado fenômeno, e, também, não considera que a construção do conhecimento científico é caracterizada pela busca por respostas para os problemas, o que favorece uma abordagem de ciência acrítica, atemporal e descontextualizada na sala de aula (ALLCHIN, 2004; CARVALHO; GIL-PÉREZ, 2011; KIPNIS, 2001; MARTINS, 2006).

Argumentar que a História da Ciência tem possibilidades didáticas para a educação científica significa que essa área do conhecimento apresenta implicações teóricas e práticas no ensino de Ciências, que vão desde a contribuição para abordar os problemas da construção do conhecimento científico podendo perpassar pela discussão da influência de aspectos sociais e culturais de uma determinada época, e chegar até a compreensão dos condicionantes das hipóteses, experimentos e argumentos científicos que foram cruciais para compreender os fenômenos do cotidiano (CARVALHO; GIL-PÉREZ, 2011; MARTINS, 1990; MARTINS; 2007; MATTHEWS, 1995).

Entretanto, estudos revelaram que essa abordagem no ensino de Ciências pode não ser garantida, se a História da Ciência ainda estiver sendo omitida em função dos resultados científicos aceitos atualmente, por meio de uma perspectiva de ciência linear e cumulativa (ALLCHIN, 2004; DUARTE, 2004; MARTINS, 2007; MATTHEWS, 1995).

Ademais, a abordagem do conhecimento científico no ensino de Ciências demanda uma preocupação com a aprendizagem de Ciências pelos estudantes (EL-HANI, 2006), o que também significa expandir essa preocupação para a formação de professores, pois:

[...] a simples consideração de elementos históricos e filosóficos na formação inicial de professores das áreas científicas - ainda que feita com qualidade - não garante a inserção desses conhecimentos nas salas de aula do ensino básico, tampouco uma reflexão mais aprofundada, por parte dos professores, do papel da HFC para o campo da didática das ciências. As principais dificuldades surgem quando pensamos na utilização da HFC para fins didáticos, ou seja, quando passamos dos cursos de formação inicial para o contexto aplicado do ensino e aprendizagem das ciências (MARTINS, 2007, p. 115).

A abordagem do conhecimento científico no ensino de Ciências é influenciada por uma variedade de conhecimentos necessários para professores de Ciências, que vão além daquele referente à matéria a ser ensinada, e inclui conhecer as inovações das pesquisas para o ensino de Ciências e saber intermediar a mobilização de habilidades de Pensamento Crítico em sala de aula (CARVALHO; GIL-PÉREZ, 2011; TENREIRO-VIEIRA; VIEIRA, 2005).

Em face dessa perspectiva, este trabalho filia-se ao entendimento de que ideias, temas, problemas e argumentos relacionados à construção do conhecimento científico em sala de aula podem ser considerados como um possível direcionamento na elaboração de materiais didáticos para o ensino de Ciências, baseados em um arcabouço de conhecimentos teóricos, que viabilizem o seu desenvolvimento (CARVALHO; GIL-PÉREZ, 2011; MARTINS, 1990).

Por isso, este estudo considera relevante a inserção de aspectos da História da Ciência no ensino de Ciências, pois, estes podem proporcionar ao estudante o discernimento de ciência, como uma atividade que pertence ao contexto social e resulta das necessidades deste, de modo a possibilitar que os conhecimentos científicos tenham significado para os estudantes, em atividades que estimulem e gerem o interesse deles para atuarem em suas resoluções 
(CARVALHO; GIL-PÉREZ，2011; MATTHEWS， 1995; OLIVEIRA; SILVA，2011; TENREIRO-VIEIRA; VIEIRA, 2005).

A abordagem da História da Ciência além de humanizar as Ciências e proporcionar o entendimento mais amplo do conhecimento científico, também pode possibilitar o desenvolvimento e aprimoramento de habilidades de Pensamento Crítico, mas, para que essa abordagem favoreça o Pensamento Crítico é preciso compreender o seu significado e a sua importância para o ensino de Ciências, para poder refletir sobre formas de articular seus aspectos dentro da dinâmica de aquisição de conhecimentos científicos (ALLCHIN, 2004; MATTHEWS, 1994, 1995, 2002; TENREIRO-VIEIRA; VIEIRA, 2000).

\section{O desenvolvimento de pensamento crítico para a tomada de decisão}

A ênfase na formação para a cidadania tem sido ressaltada por diversas pesquisas no ensino de Ciências, que se propõem a defendê-la como uma finalidade para a educação científica, visando o desenvolvimento da tomada de decisão e a resolução de problemas pelos estudantes (CACHAPUZ et al., 2005; CARVALHO; GIL-PÉREZ, 2011; FREIRE, 2007; TENREIRO-VIEIRA; VIEIRA, 2000, 2020).

Contudo, essas considerações carregam a necessidade de explicitar e delinear essa formação para a cidadania que é defendida, pois pode estar condicionada a uma pluralidade de significados e de objetivos para o ensino de Ciências tornando mais complexa a tarefa inovadora de apresentar propostas de ensino com esta finalidade (CACHAPUZ et al., 2005; MOURA; GUERRA; 2016).

Para tal é preciso refletir, debater e propor ações emergentes para uma educação científica, que exige sua realização no contexto da contemporaneidade, uma vez que essa formação para a cidadania pode ser entendida também como uma perspectiva futura (CACHAPUZ et al., 2005). Isso decorre da imprevisibilidade que caracteriza as necessidades da sociedade na contemporaneidade e em instâncias futuras, sendo imprescindível fomentar propostas de ensino para uma formação integral, que crie oportunidades de aprendizagem para os estudantes atuarem na realidade em que vivem (TENREIRO-VIEIRA; VIEIRA, 2021). Essa formação também envolve considerar que:

\footnotetext{
Continuar aprendendo depois que é dada por encerrada a etapa da educação básica, é fundamental em um mundo de mudanças contínuas. Dotar o educando de instrumentos que o permitam continuar aprendendo não é uma tarefa simples, mas todo o contexto do ensino (ambiente escolar, atores, materiais...) pode contribuir para desenvolver essa capacidade nos jovens (FREIRE, 2007, p. 11).
}

Essas são necessidades emergentes, do contexto contemporâneo na educação científica, que exigem o desenvolvimento da autonomia intelectual dos estudantes e de habilidades cognitivas, a partir das quais seja possível estimular os estudantes a analisarem as informações científicas, participarem das atividades no coletivo e atuarem na proposição de solução para os problemas das atividades propostas (FREIRE, 2007; LIPMAN, 1989; TENREIRO-VIEIRA; VIEIRA, 2000). Esses aspectos são fundamentais e têm se tornado destaque, por considerarem um processo de ensino fundamentado no desenvolvimento de habilidades cognitivas, relacionadas ao uso consciente do Pensamento Crítico pelos estudantes (FREIRE, 2007; LIPMAN, 1988; TENREIRO-VIEIRA; VIEIRA, 2000).

O pensamento crítico tem sido estudado por pesquisadores de áreas diversas, a exemplo da filosofia com Ennis (1985), que tem sido citado como teórico influente de pesquisas sobre o desenvolvimento de habilidades cognitivas dos estudantes, e seus estudos tem servido como 
base para diversos trabalhos sistematizados por Tenreiro-Vieira e Vieira (2000, 2005, 2006, 2015, 2019, 2020, 2021), em proposições para o ensino e a aprendizagem de Ciências.

Mas, o que significa o pensamento crítico e qual sua relação com a educação científica? Esses são questionamentos iniciais que podem ser considerados basilares para a compreensão de sua importância nas práticas do ensino de Ciências, sendo uma justificativa para este estudo.

O pensamento crítico apresenta diversas definições, dentre elas, no âmbito dos estudos da psicologia cognitiva, Halpern (1999) atribui que o Pensamento Crítico se refere ao uso de habilidades ou estratégias cognitivas, que possibilitam aumentar as chances de se obter um resultado desejável, sendo caracterizado como objetivo, racional e direcionado para uma meta. Esse pensamento está envolvido na resolução de problemas, na construção de inferências, na formulação de hipóteses, no cálculo de probabilidades e na tomada de decisões, essas habilidades, quando aprendidas pelos estudantes e aplicadas adequadamente, possibilitam torná-los pensadores melhores (FREIRE, 2007; HALPERN, 1999).

Se essas concepções forem aprofundadas, o pensamento crítico poderá ser considerado como um objetivo fundamental da educação científica, uma vez que esse processo envolve a formação de estudantes para que aprendam a pensar por si mesmo, isso justifica o porquê desse pensamento não ser arbitrário, pois demanda ações de intervenção consciente (CACHAPUZ et al., 2005; LIPMAN, 1988; SIEGEL, 1989; TENREIRO-VIEIRA; VIEIRA, 2000). Desse modo:

Formar o educando como pessoa humana implica em discutir valores, atitudes e normas próprias de uma sociedade. Tudo isso contribui para a formação da cidadania. A preparação para o trabalho requer além de normas, valores e atitudes, desenvolvimento interpessoal e aprimoramento de conhecimentos técnicos (FREIRE, 2007, p. 11).

Contudo, a forma como o ensino tem sido abordado pode não favorecer a sua interface com a dinâmica social, pois pode estar sendo demarcado pela excessiva explanação de conteúdos científicos, caracterizando-se por uma ausência de propostas que viabilizem a mobilização e o desenvolvimento de atitudes, habilidades de pensamento, de análise e interpretação de informações científicas e de argumentação (FREIRE, 2007; TENREIROVIEIRA; VIEIRA, 2000).

Um ensino que objetive o desenvolvimento dos estudantes para atuar no contexto em que se vive exige propostas que ressaltem o desenvolvimento de habilidades cognitivas relacionadas ao Pensamento Crítico dos estudantes, de modo atrelado a abordagem de conhecimentos científicos, para dar sentido ao processo de aprendizagem de Ciências (MATTHEWS, 1995; TENREIRO-VIEIRA; VIEIRA, 2000, 2005).

Neste estudo, considera-se que as habilidades de Pensamento Crítico estão atreladas ao desenvolvimento de ações provenientes do âmbito cognitivo, que possibilitem a aquisição e o uso do conhecimento científico para a tomada de decisão na resolução de problemas que envolvem ciência, e que a abordagem do conhecimento científico pode ser realizada pela História da Ciência (TENREIRO-VIEIRA; VIEIRA, 2000, 2019).

Para tanto, este estudo teórico tem o objetivo de inserir aproximações entre a História da Ciência e o Pensamento Crítico no ensino de Ciências. Essas aproximações se constituem em elementos de um modelo teórico, que busca apresentar articulações diretas e indiretas dessas perspectivas, por meio da consideração de seus objetivos para o ensino de Ciências, e, do seu ponto de convergência pensando na proposição didática.

\section{Processo de articulação das perspectivas teóricas}


As discussões sobre a educação científica, suscitadas por Cachapuz e colaboradores (2005), ressaltam que esse processo demanda pensar em propostas atuais para a sua implementação no ensino de Ciências. Por conseguinte, abordar a educação científica torna-se complexo, em face da amplitude e variedade de ações para esse propósito, mas em se tratando do ensino para a aprendizagem dos estudantes, o conhecimento para a tomada de decisão pode ser considerado como um viés fundamental nesse processo (CACHAPUZ et al., 2005).

Nesse sentido é discutida a necessidade de o ensino de Ciências ser compreendido como uma formação geral para os estudantes, e, a reflexão sobre seus objetivos e propósitos pode conduzir à decisões no processo de ensino de Ciências tendo a História da Ciência como uma abordagem relevante para a formação crítica (CACHAPUZ et al., 2005; MARTINS, 2006; MATTHEWS, 1995).

Considerando essas premissas, este trabalho advém de uma pesquisa que enfatiza a possibilidade dos pressupostos da História da Ciência e do Pensamento Crítico constituírem abordagens centralizadas no conhecimento para a tomada de decisão (CACHAPUZ et al., 2005; MARTINS, 2006; MATTHEWS, 1995; TENREIRO-VIEIRA; VIEIRA, 2000, 2005, 2019). Nesse sentido são apresentados elementos que culminaram na articulação teórica de seus objetivos para o ensino de Ciências, porém, isso não significa a redução da História da Ciência e do Pensamento Crítico como se fossem meros instrumentos, ao contrário dessas concepções que reduzem sua complexidade, neste trabalho, elas são consideradas como perspectivas para a abordagem teórica, com o intuito de contribuir para a projeção de propostas didáticas (CARDOSO, 2021).

Desse modo, este estudo procura inserir-se no âmbito da pesquisa qualitativa, por compreender que a proposição de materiais didáticos depende da delimitação do conjunto de conhecimentos teóricos que são a base para o seu desenvolvimento (COUTINHO, 2016).

A justificativa para a realização dessa pesquisa se deu durante um mestrado acadêmico (concluído em 2021), ao verificar a necessidade de contemplar uma abordagem de ensino baseada na História da Ciência e orientada para o desenvolvimento de capacidades de Pensamento Crítico no ensino de Ciências (CARDOSO, 2021). Durante essa pesquisa, na revisão da literatura foi possível verificar que os trabalhos que objetivavam o desenvolvimento do Pensamento Crítico em abordagens de História da Ciência, não investigaram o desenvolvimento de habilidades cognitivas dos estudantes nas propostas didáticas e não apresentaram nenhuma correlação entre os seus objetivos para o ensino de Ciências (CARDOSO, 2021).

Ao considerar a importância dessa correlação em abordagens de ensino, este estudo procurou em referenciais que abordam aspectos de História da Ciência e naqueles que investigam e debatem sobre o Pensamento Crítico, os seus objetivos para o ensino de Ciências, a fim de realizar as aproximações teóricas (CARDOSO, 2021). Esse exercício de articulação foi realizado a fim de responder a questão de pesquisa "Dos referenciais selecionados, quais dos seus objetivos para o ensino de Ciências, que podem possibilitar uma aproximação teórica de História da Ciência e do Pensamento Crítico?".

De acordo com as fases da Análise de Conteúdo (BARDIN, 2011), a seleção de fontes foi realizada com base na leitura e exploração de materiais de História da Ciência, em busca do que esses referenciais relatam sobre a formação crítica dos estudantes, ou, se as concepções apresentadas sobre o ensino de Ciências tinham como objetivo o desenvolvimento de habilidades de Pensamento Crítico, a exemplo de Matthews (1994, 1995, 2002), teórico que deixa claro sua tendência em defender a História da Ciência como viés para abordar o conhecimento científico, e, como possibilidade didática para o desenvolvimento de habilidades de Pensamento Crítico no ensino de Ciências. 
Cabe salientar, que o estudo não procurou esgotar a totalidade de pesquisas no ensino de Ciências que abordam a História da Ciência e o Pensamento Crítico, pois, se compreende a impossibilidade de fazê-lo em face da já explicitada complexidade dessas áreas, sendo também uma possibilidade de incentivar continuações e inovações de pesquisas que podem surgir.

Para a seleção de referenciais sobre o Pensamento Crítico, esse processo considerou autores que sistematizam o ensino do Pensamento Crítico na educação científica, a exemplo de Tenreiro-Vieira e Vieira (2000, 2005) e Freire (2007). Apesar de Halpern (1999) demandar estudos provenientes de investigações da área da psicologia cognitiva, este também foi um referencial investigado, pois, se propõe a debruçar seus estudos sobre a influência dessa perspectiva em estudantes, a fim de torná-los pensadores melhores.

Com a seleção das considerações de diferentes autores foi possível destacar os objetivos para o ensino de Ciências no âmbito da História da Ciência e do Pensamento Crítico e tecer as aproximações teóricas entre eles, sendo considerados apenas aqueles que possibilitaram uma correlação.

Baseado nos pressupostos de Bardin (2011), para diferenciar os referenciais citados como aporte teórico deste trabalho dos referenciais que foram selecionados e analisados nas aproximações teóricas, este estudo considerou a sua codificação conforme é apresentada no Quadro 1 a seguir:

Quadro 1 - Objetivos de HC e PC para o ensino de Ciências.

\begin{tabular}{|c|c|}
\hline Objetivos & Codificação dos referenciais analisados de HC e PC \\
\hline $\begin{array}{l}\text { 1- Compreender a ciência como uma } \\
\text { atividade desenvolvida no contexto social }\end{array}$ & $\begin{array}{c}\text { Forato_Pietrocola_Martins_2011, Freire_2007, } \\
\text { Martins_2006, Matthews_1995, Sequeira_Leite_1988 e } \\
\text { Tenreiro-Vieira_Vieira_2000; }\end{array}$ \\
\hline $\begin{array}{l}\text { 2- Compreender e interpretar aspectos da } \\
\text { ciência de uma forma mais ampla }\end{array}$ & $\begin{array}{l}\text { Forato_Pietrocola_Martins_2012, Freire_2007, } \\
\text { Gagliardi_1988, Matthews_1994 e Matthews_1995; }\end{array}$ \\
\hline $\begin{array}{l}\text { 3- Formação crítica para atuar na resolução } \\
\text { de problemas que envolvem aspectos } \\
\text { científicos no contexto social }\end{array}$ & $\begin{array}{c}\text { Forato_Pietrocola_Martins_2012, Freire_2007, Freire- } \\
\text { Junior_2002, Hidalgo_Lorencini-Junior_2016, } \\
\text { Martins_1990, Matthews_1994, Matthews_2002 e } \\
\text { Tenreiro-Vieira_Vieira_2000; }\end{array}$ \\
\hline $\begin{array}{l}\text { 4- Analisar adequadamente informações } \\
\text { que envolvem questões científicas no } \\
\text { contexto social }\end{array}$ & $\begin{array}{c}\text { Halpern_1999, Matthews_1994, Sequeira_Leite_1988 e } \\
\text { Tenreiro-Vieira_Vieira_2000; }\end{array}$ \\
\hline $\begin{array}{l}\text { 5- Problematizar aspectos científicos de } \\
\text { modo a interagir com os outros para a } \\
\text { resolução de problemas }\end{array}$ & $\begin{array}{l}\text { Forato_Pietrocola_Martins_2012, Freire_2007, } \\
\text { Matthews_1994, Matthews_1995, Peduzzi_2005 e } \\
\text { Tenreiro-Vieira_Vieira_2000; }\end{array}$ \\
\hline $\begin{array}{l}\text { 6- Diferenciar afirmações e argumentos } \\
\text { baseados em dados científicos dos que não } \\
\text { são. }\end{array}$ & $\begin{array}{c}\text { Matthews_1994, Matthews_1995, Peduzzi_2005 e } \\
\text { Tenreiro-Vieira e Vieira_2000; }\end{array}$ \\
\hline
\end{tabular}

Para realizar a síntese desses objetivos em temas gerais, conforme apresentados no Quadro 1, foi preciso considerar os trechos que significavam as pretensões para o ensino de Ciências de cada referencial teórico selecionado, e, depois buscou-se correlacionar aspectos em 
comum salientados pelos autores que podiam ser aproximados, conforme os pressupostos da Análise de Conteúdo (BARDIN, 2011; COUTINHO, 2016).

\section{Modelo de aproximações e sua ênfase no conhecimento para a tomada de decisão}

As aproximações teóricas foram realizadas em função da necessidade de compreender quais aspectos estão relacionados diretamente com a dimensão pedagógica, ou seja, quais deles podem tornar possível a sua proposição em materiais didáticos, que ressaltem a perspectiva histórica da construção do conhecimento científico para o desenvolvimento de habilidades de Pensamento Crítico (CARDOSO, 2021).

Isso possibilitou compreender que essa busca por objetivos para o ensino de Ciências tem como eixo central uma ênfase no conhecimento para a tomada de decisão, o que explicita e justifica a correlação intrínseca que foi possível salientar pelas bases teóricas da História da Ciência e do pensamento crítico, e como essas podem ser orientadoras de abordagens didáticas. Esses excertos partem da clarificação realizada por esta pesquisa, ao depreender dos estudos de Cachapuz e colaboradores (2005), Cardoso (2021), Carvalho e Gil-Pérez (2011) e TenreiroVieira e Vieira (2019), a dificuldade em se realizar essas projeções nas propostas de ensino sem um fio condutor, e, a necessidade de articular as perspectivas teóricas, de forma a estabelecer uma base para possíveis projeções orientadas explicitamente para esse propósito.

Para tanto, a interface das perspectivas teóricas em objetivos para o ensino de Ciências, que foi salientada no Quadro 1, possibilitou classificá-los como correlações explícitas e implícitas das aproximações teóricas, pois foi possível observar correspondências ou similitudes entre as concepções apresentadas pelos referenciais selecionados, como pode ser observado na Figura 1, que é apresentada a seguir:

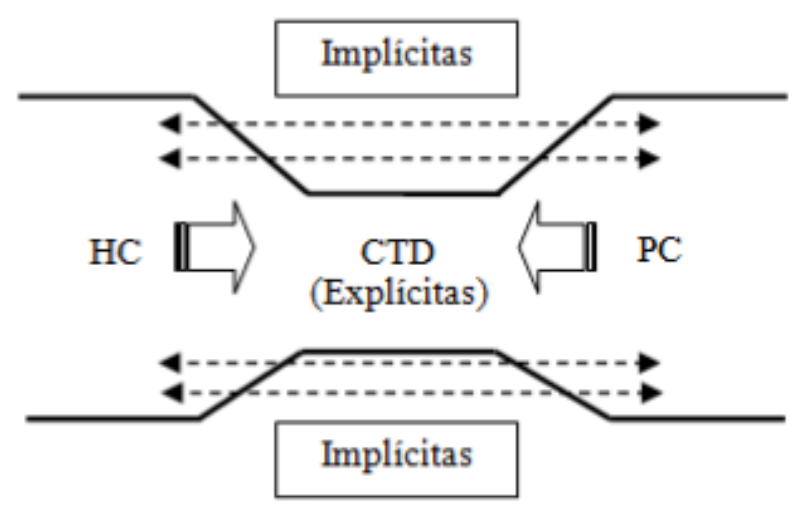

Figura 1 - Modelo teórico CTD de aproximações HC-PC Fonte: Cardoso (2021, p. 62)

No modelo teórico, apresentado na Figura 1, o Conhecimento para a Tomada de Decisão (CTD) é considerado como o aspecto central, porque o ponto de convergência das aproximações teóricas, correlacionadas a partir das perspectivas da História da Ciência (HC) e do Pensamento Crítico (PC), visa direta e explicitamente o ensino de Ciências, pois, seus objetivos se projetam em função do aspecto pedagógico. Desse modo, essas correlações teóricas dos objetivos de HCPC foram denominadas de dimensões explícitas ou CTD, sendo representadas no modelo teórico da Figura 1 pelas duas setas que convergem de História da Ciência e do Pensamento 
Crítico para a dimensão do Conhecimento para a Tomada de Decisão, campo que corresponde aos objetivos quatro, cinco e seis $(4,5$ e 6$)$, que foram apresentados no Quadro 1 (CARDOSO, 2021).

Na proposta do modelo teórico (Figura 1), também aparecem dimensões implícitas, nas quais as convergências entre HC e PC são indiretas, pois, alguns dos seus objetivos não estão relacionados diretamente com o conhecimento para a tomada de decisão, ou seja, nem todos direcionam para a prática pedagógica, como pode ser visto na Figura 1 pelas setas tracejadas e fora da dimensão CTD, já que os seus objetivos não convergiam para esse aspecto central. Essas dimensões implícitas são constituídas pelos objetivos um, dois e três (1, 2 e 3), apresentados no Quadro 1 (CARDOSO, 2021).

Cabe ressaltar que o modelo teórico da Figura 1, não garante a abordagem adequada de episódios históricos e de habilidades cognitivas, pois, esse modelo também demanda preocuparse com a maneira como poderão ser planejadas essas dimensões CTD na proposta didática, qual a temática a ser abordada, assim como também depende do episódio histórico da construção do conhecimento científico a ser selecionado e quais as atividades que possam salientar o desenvolvimento das habilidades cognitivas, para que viabilize a sua proposição de forma intencional (CARDOSO, 2021; MATTHEWS, 1995; TENREIRO-VIEIRA; VIEIRA, 2005). Portanto, essas articulações permitiram a elaboração de um modelo teórico, que pode ser utilizado para realizar uma aproximação entre a História da Ciência e o Pensamento Crítico na proposição de materiais didáticos (CARDOSO, 2021).

\section{As dimensões do modelo teórico e suas implicações para o ensino de Ciências}

A discussão sobre as correlações implícitas e explícitas demarca a compreensão do motivo pelo qual essas dimensões são consideradas neste trabalho, e, a pertinência de seus aspectos em função do referencial teórico selecionado, destacado no Quadro 1 e que são apresentadas a seguir: ${ }^{1}$

\section{a) dimensões implícitas}

São as correlações aproximadas de História da Ciência e do Pensamento Crítico, que comportam os objetivos que, mesmo sendo sobre o ensino de Ciências, não apresentam ações que indiquem diretamente o uso do conhecimento para a tomada de decisão, mas se referem à formação crítica, a compreensão ampla do conhecimento científico e da ciência como uma atividade desenvolvida no contexto social caracterizando-a como uma atividade humana. Do Quadro 1 são considerados como dimensões implícitas ou indiretas os seguintes objetivos:

1- Compreender a ciência como uma atividade desenvolvida no contexto social, esse objetivo enfatiza que estudos adequados de episódios históricos possibilitam o entendimento da ciência como um processo social e gradativo da construção do conhecimento científico, e, as ideias que vão sendo construídas e aperfeiçoadas se desenvolvem mediante debates e divergências, como ressaltado por Martins (2006) no recorte apresentado a seguir:

O estudo adequado de alguns episódios históricos também permite perceber o processo social (coletivo) e gradativo de construção do conhecimento científico, permitindo formar uma visão mais correta da real natureza da ciência, seus procedimentos e suas limitações - o que contribui para a formação de um espírito crítico e desmitificação do conhecimento científico, sem, no entanto, negar seu valor

\footnotetext{
${ }^{1}$ Notar que este tópico apresenta as aproximações teóricas analisadas, identificadas pela codificação do Quadro 1 e sua discussão em função da literatura de base.
} 
(Ibidem, p. 18)

Para empreender um processo de educação formal, no qual as preocupações enfatizam a formação de uma visão mais crítica e adequada tanto da ciência como de sua atividade científica é preciso compreender que essas intrínsecas relações estão nos complexos sistemas que regem a realidade cotidiana e possibilitar o desenvolvimento do Pensamento Crítico dos estudantes em meio à dinâmica de suas atividades (KIPNIS, 2001; MATTHEWS, 1995; TENREIRO-VIEIRA; VIEIRA, 2000).

Esses pressupostos conduzem a reflexão sobre como a abordagem da construção do conhecimento científico pode ser realizada em propostas do ensino de Ciências por meio da História da Ciência, como pode verificado no recorte apresentado a seguir:

\footnotetext{
Um professor de ciências com conhecimento de HFS pode auxiliar os estudantes a compreender exatamente como a ciência apreende, e não apreende, o mundo real, vivido e subjetivo. Porém, o mais comum é que o estudante fique sujeito à infeliz escolha entre renunciar ao seu próprio mundo por ser uma fantasia, ou renunciar ao mundo da ciência pela mesma razão (MATTHEWS, 1995, p. 22)
}

Para desmitificar essa concepção de conhecimento científico como verdade universal, esse processo pode ser abordado no ensino de Ciências como uma perspectiva que se desenvolve no contexto social, pois, a ciência se propõe a compreender os fenômenos do mundo em que se vive, porém, esta deve ser uma tarefa de apresentação da ciência como uma atividade humana, ao procurar aproximá-la de outras atividades desenvolvidas no contexto social, além de viabilizar a construção de conhecimentos em sala de aula e o desenvolvimento de habilidades de Pensamento Crítico em meio a exercícios de raciocínio lógico (CARVALHO; GIL-PÉREZ, 2011; KIPNIS, 2001; MATTHEWS, 1995). Nesse sentido é possível verificar o exposto acima discutido no recorte a seguir:

A compreensão da ciência como uma atividade humana, historicamente construída, imersa no contexto cultural de cada época e de cada povo, e não como uma construção puramente racional, desenvolvida por um suposto "método científico" único e universal a partir apenas de observações, experimentos, deduções e induções logicamente fundados (MARTINS, 2011, p. 6)

Por isso, Forato_Pietrocola_Martins_2011, também atribuem que para esse processo acontecer é preciso romper com visões distorcidas sobre a ciência e possibilitar a discussão dessa atividade como caracterizada por dilemas profissionais, sendo um conhecimento falível, que pode ser contestado e influenciado por fatores extra-científicos, o que permite viabilizar a promoção do Pensamento Crítico, que em sua essência é um pensamento reflexivo (TENREIRO-VIEIRA; VIEIRA, 2000, 2005).

Nesse sentido, para Allchin (2004), Cachapuz e colaboradores (2005), Duarte (2004) e Tenreiro-Vieira e Vieira (2000), a abordagem do conhecimento científico como uma empreitada desenvolvida no contexto social pode viabilizar a construção de significados pelos estudantes, que podem começar a entender a ciência não mais como um conhecimento distante de sua realidade, mas sim, como uma perspectiva para se entender o mundo real.

2- Compreender e interpretar aspectos da ciência de uma forma mais ampla, no sentido de conhecer e compreender não apenas os conteúdos científicos, mas também, seus limites e validades, como salienta o estudo de Forato Pietrocola Martins (2012). Desse modo, como um referencial que discute sobre abordagens para o ensino do pensamento crítico, a perspectiva teórica de Freire (2007) atribui que nesse processo é preciso ir além das explicações simplistas, 
na resolução de problemas é preciso realizar observações e apresentar justificativas, de modo que sejam desenvolvidas e apresentadas perspectivas múltiplas sobre os problemas ou situações, abordadas no processo de ensino, que podem simular situações da vida cotidiana. Assim é possível observar do recorte retirado a seguir que:

Em Ciências - da natureza ou humanas - o senso comum convive com o pensamento científico em questões cotidianas, mas não deve ser o balizador de opiniões em questões mais elaboradas. As pessoas que se deixam guiar apenas pelos pensamentos simplistas, dificilmente conseguirão elaborar um pensamento crítico (Ibidem, p. 27)

Como contraposição a tendência de considerar que o conhecimento científico deve suplantar os conhecimentos espontâneos dos estudantes por explicar melhor uma situação, problema ou fenômeno estudado, assim como também é realizado com teorias científicas, a História da Ciência pode auxiliar na empreitada de apresentar uma visão mais plural da ciência no ensino, pois pode ser um viés para perspectivas tanto teóricas quanto práticas do ensino de Ciências (CARVALHO; GIL-PÉREZ, 2011; KIPNIS, 2001; MATTHEWS, 1995).

Nesse processo, considera-se que para os estudantes se desenvolverem na resolução de problemas, se faz necessário a mobilização de recursos nos quais os estudantes possam empreender uma compreensão ampla do conhecimento científico, pois somente dessa maneira será possível os estudantes atuarem e intervirem de forma consciente, sendo preciso um mínimo de formação científica a ser fornecida para eles (CACHAPUZ et al., 2005; CARVALHO; GILPÉREZ, 2011). Sobre a abordagem da construção do conhecimento científico no ensino de Ciências é possível observar do recorte apresentado a seguir que:

Uma parte da contribuição da HFS para o ensino de ciências é conectar tópicos em determinadas disciplinas científicas, conectar as disciplinas da ciência entre si, conectar as ciências em geral com matemática, filosofia, literatura, psicologia, história, tecnologia, comércio e teologia. E, finalmente, exibir as interconexões da ciência e da cultura - artes, ética, religião, política - de maneira mais ampla (MATTHEWS, 1994, p. 5. Tradução nossa).

Isso significa que a abordagem do conhecimento científico pode ser realizada de forma mais ampla, como pressupõe a perspectiva teórica de Gagliardi (1988, p. 2. Tradução nossa): "Para introduzir nas aulas discussões sobre a produção, apropriação e controle de conhecimentos a nível individual e social", ou seja, ir além das explicações simplistas, de modo a proporcionar que os estudantes apresentem explicações que estão para além daquilo que está descrito, de modo a interpretar a situação como um todo, como salienta a perspectiva teórica apresentada em Freire (2007).

3- Formação crítica para atuar na resolução de problemas que envolvem aspectos científicos no contexto social. Na perspectiva teórica de Matthews (2002), essa formação está relacionada ao desenvolvimento de aptidões de caráter informativo, crítico e moral atrelada às capacidades de Pensamento Crítico no âmbito pessoal e social, algo que também foi possível destacar do referencial sobre Pensamento Crítico de Tenreiro-Vieira (2000), que enfatiza que esse processo envolve saber atuar no cotidiano para a resolução de problemas.

Então, para compreender a ciência, é necessária uma formação para o desenvolvimento da capacidade crítica e de abertura de pensamento, para questionar as informações sobre aspectos que envolvem a ciência no contexto social, como foi possível evidenciar na perspectiva teórica sobre História da Ciência de Hidalgo Lorencini Junior (2016) e Martins (1990).

Nesse sentido, o ensino para o desenvolvimento do pensamento crítico tornou-se cada vez mais fundamental e necessário em face da constatação de que é essencial e deve ser a base 
para uma formação que valorize o desenvolvimento de estudantes capazes de lidar e saber atuar frente às mudanças constantes e complexas dos sistemas que regem a dinâmica da sociedade atual, pois, sem essa formação os estudantes estarão condicionados a serem conduzidos pelo sistema e por pessoas (FREIRE, 2007; TENREIRO-VIEIRA; VIEIRA, 2000, 2019). Essa formação crítica tem sido atrelada com o desejo de fomentar uma formação para o desenvolvimento da cidadania, como no recorte a seguir:

\footnotetext{
O desafio é formar o cidadão de modo que ele possa ter uma relação crítica com algo que é parte intrínseca do processo de humanização e civilização, mas cuja função e papel precisam se tornar parte das preocupações comuns aos humanos [...]. Os rumos da ciência e da tecnologia na contemporaneidade precisam integrar esta agenda de preocupações, mas para isso é preciso formar o cidadão com os recursos intelectuais e morais necessários a esta integração (FREIRE JUNIOR, 2002, p. 20).
}

Esse é um dos aspectos considerados como finalidade da educação científica, por referenciais que pesquisam sobre metodologias para um ensino baseado no desenvolvimento do Pensamento Crítico de estudantes, pois objetivam que essa abordagem não pode acontecer de forma dissociada, mas sim, pela mobilização dos recursos intelectuais dos estudantes, o que inclui a aquisição de conhecimentos científicos em um processo que proponha condições para que desenvolvam habilidades cognitivas relacionadas ao Pensamento Crítico, e, a História da Ciência pode ser uma abordagem adequada para tal finalidade (LIPMAN, 1988; MATTHEWS, 1995; TENREIRO-VIEIRA; VIEIRA, 2019). Como pode ser observado no recorte a seguir:

[...] o ensino de Ciências precisa ser compreendido como algo que contribui para a formação geral dos alunos [...] a educação deve estar preocupada primordialmente em desenvolver a compreensão, mediante uma iniciação nas tradições importante do pensamento, e em desenvolver a aptidão para o pensamento claro, analítico e crítico [...] e, finalmente, apropriar-se de forma crítica do que está aprendendo [...] (MATTHEWS, 2002, p. 33-34).

Essa formação geral está relacionada a um processo de reflexão sobre práticas de ensino que proporcionem uma formação científica, pois, sem o mínimo dessa formação para atuar com os conhecimentos científicos no contexto social, os estudantes não serão estimulados a aprender a ter responsabilidade por seus pensamentos e nem saberão participar na tomada de decisão ou resolução de problemas (CACHAPUZ et al., 2005; LIPMAN, 1988).

\section{b) Dimensões explícitas}

Essas dimensões se referem aos objetivos (apresentados no Quadro 1) que representam ações que retratam o uso do conhecimento para a tomada de decisão, de modo a viabilizar a sua projeção em propostas de ensino de Ciências, com a finalidade de apresentar uma abordagem do conhecimento científico e do desenvolvimento de atividades para uma possível mobilização de habilidades do Pensamento Crítico, conforme apresentado a seguir:

4- Analisar adequadamente informações que envolvem questões ou aspectos científicos relacionados com o contexto social, de modo que possam ser apresentados posicionamentos em face dessa análise. Nesse aspecto, apresenta-se a compreensão do conhecimento científico como sendo necessária para enfatizar a participação social na tomada de decisões dos problemas científicos, como pode ser observado nas perspectivas teóricas de (HALPERN, 1999; MATTHEWS, 1994; TENREIRO-VIEIRA, 2000).

Para tal, a análise das informações recebidas e compartilhadas demanda a capacidade de Pensamento Crítico de avaliar a credibilidade de uma fonte de informação, de modo a 
possibilitar o julgamento das ideias, como ressaltado na perspectiva teórica de Halpern (1999) e de Tenreiro-Vieira Vieira (2000). Nesse sentido, o recorte a seguir atribui que:

\begin{abstract}
A História da Ciência pode assim contribuir para que os alunos se sintam mais à vontade não só para apresentar ao professor e aos colegas as suas próprias ideias de mundo mas também para as discutir e avaliar, melhorando a comunicação na sala de aula [...] permitindo aos alunos constatar como os modelos em ciência são modificados e adaptados a novos dados (experimentais ou teóricos), descobertas e progressos de outros ramos do saber, como os aspectos sociais, econômicos e políticos e até a crença pessoal (LEITE, 1988, p. 7)
\end{abstract}

Isso possibilita considerar que o ensino de Ciências demanda a proposição de atividades nas quais tenham como aspecto central a análise de informações científicas, que possuam significado para os estudantes, ou seja, que estejam contextualizadas com aspectos sociais, pois, para que os estudantes possam apresentar e discutir suas concepções de mundo com seus colegas, isso exige uma dinâmica voltada para o julgamento de informações, no qual o conhecimento deve ser utilizado na tomada de decisão (CACHAPUZ et al., 2005; HALPERN, 1999; MATTHEWS, 1995; TENREIRO-VIEIRA; VIEIRA, 2000).

Por isso, essa abordagem do conhecimento científico permite considerar em sala de aula a ciência como um conhecimento essencial para compreender o mundo em que se vive (GURGEL, 2020; KIPNIS, 2001), como pode ser evidenciado no recorte a seguir sobre a ciência como uma atividade que:

Trecho retirado de Matthews_1994_p.5-10: [...] forneceu, ainda que vacilante, uma contra-influência às inclinações naturais das pessoas para julgar as circunstâncias em termos de seu próprio interesse. Isso instiga uma preocupação com evidências e com ideias julgadas não pelo interesse pessoal ou social, mas pela maneira como o mundo é [...]. O ensino de Ciências é aprimorado se os alunos souberem o significado dos termos que estão usando e se puderem pensar criticamente sobre textos, relatórios e sua própria atividade científica [...] desenhar gráficos, elaborar hipóteses, pensar em situações de controle, localizar erros [...] (MATTHEWS, 1994, p. 5-10. Tradução nossa).

Essas demandas surgem a partir da compreensão de que as mudanças que ocorrem na sociedade contemporânea também influenciam metodologias e abordagens no ensino de Ciências, pois, estas precisam se adaptar as necessidades da formação científica, que passa a exigir cada vez mais, que os estudantes sejam formados para atuarem na dinamicidade das complexas relações estabelecidas nesse meio em que vivem, e, para que saibam intervir através de suas habilidades cognitivas, o que implica favorecer a autonomia intelectual, por meio de atividades que estimulem a investigação científica (EL-HANI, 2006; HALPERN, 1999; LIPMAN, 1988; TENREIRO-VIEIRA; VIEIRA, 2000, 2005, 2019).

5- Problematizar aspectos científicos de modo a interagir com os outros, para a resolução de problemas. Esse objetivo compreende a importância de ações para debater ideias sobre a construção do conhecimento científico discutindo e avaliando essas ideias com os outros e possibilitando desenvolver uma atitude questionadora, como salientado nas perspectivas teóricas (FORATO; PIETROCOLA; MARTINS, 2012; MATTHEWS, 1994, 1995; TENREIRO-VIEIRA VIEIRA, 2000).

Nesse sentido, é preciso ressaltar que antes dos estudantes tomarem consciência e decidir sobre algo, todo esse processo é influenciado pelas interações deles com a família, os colegas, os professores, os meios de comunicação, dentre outras influências, então os estudantes precisam estar preparados para lidar com essas influências de forma autônoma, consciente e 
para que saibam resolver os problemas que cercam uma determinada situação, de forma racional, ou seja, motivado por razões adequadas, principalmente quando esses problemas envolvem os conhecimentos científicos (FREIRE, 2007; LIPMAN, 1988; SIEGEL, 1989). Para tanto, a pergunta que surge é: como desenvolver atividades para tal objetivo?

A resposta para tal pergunta está inicialmente na compreensão do que é preciso para integrar e conceder significado a esse processo, para que não seja considerado como uma simples e corriqueira realização de mais uma atividade (EL-HANI, 2006; MATTHEWS, 1995). Mais especificamente, este estudo está se referindo a projeção da problematização do conhecimento científico para a resolução de problemas, como salienta o recorte a seguir:

Tratar-se-ia de casos [...] [que] seriam controvérsias fictícias sobre decisões tecnocientíficas verdadeiras, ainda que não reais. Nestes casos, as polêmicas são abertas (como nos casos reais), porém, bem definidas (como nos casos históricos). Este tipo de caso permite relacionar o lado lúdico aos planos da realidade e da ficção. [...] Queremos desenvolver nos alunos um espírito crítico, de considerar diferentes pontos de vista, de debater e apresentar argumentos fundamentados, de desenvolver um pensamento autônomo e de despertar a curiosidade intelectual (FREIRE, 2007, p. 49-50)

Desse modo, a dimensão da problematização pode exigir atividades nas quais os estudantes identifiquem ou elaborem critérios para avaliar possíveis soluções, de modo a centralizar no assunto ou problema que está em causa, assim como exige também que os estudantes participem de atividades que estimulem a formulação de hipóteses, o estudo e a proposição de definições, a proposição de argumentos para estabelecer soluções para um problema, e, que possam atuar e intervir nas situações com base nas interações com o coletivo (TENREIRO-VIEIRA; VIEIRA, 2000, 2005). Nesse sentido, no recorte a seguir é possível observar que nesse processo espera-se que os estudantes:

[...] considerem algumas das questões intelectuais que estão em jogo; espera-se que considerem o fato de que há perguntas a serem feitas e que comecem a refletir não somente sobre as respostas para essas perguntas, mas, sobretudo, sobre quais as respostas válidas e que tipos de evidências poderiam sustentar essas respostas (MATTHEWS, 1995, p.5).

Também é preciso compreender que a problematização do conhecimento deve considerar as concepções espontâneas que os estudantes apresentam, e que podem orientar a abordagem de episódios históricos, pois, alguns pesquisadores acreditam que podem ser correlacionadas aos conhecimentos já debatidos e abandonados, para proporcionar o debate, o questionamento e a reflexão dos problemas que envolvem a construção do conhecimento científico (CARVALHO; GIL-PÉREZ, 2011; MATTHEWS, 1995; PEDUZZI, 2005).

6- Diferenciar afirmações e argumentos baseados em dados científicos dos que não são científicos. Esse aspecto está relacionado à compreensão do conhecimento na análise de argumentos, a fim de diferenciá-los e procurar as conclusões nas afirmações ditas científicas, algo que é relevante para a projeção de propostas a partir da abordagem histórica, de modo que possa viabilizar o desenvolvimento do pensamento crítico utilizando aspectos do conhecimento científico para a tomada de decisão, como enfatizado nas perspectivas teóricas de Matthews (1995) e Tenreiro-Vieira Vieira (2000).

Isso demanda preocupar-se com a simplificação adequada dos episódios históricos para realizar essa abordagem, pois, como salienta o recorte a seguir: 
Realmente, uma dada seleção histórica da evolução dos assuntos de um corpo específico de conhecimentos, em qualquer situação, será sempre um subconjunto do real e intrincado emaranhado de relações que lhe conferiram dinamicidade. E isto não é exceção no sistema educacional, onde as disciplinas que estruturam qualquer currículo lidam com cargas horárias limitadas (PEDUZZI, 2005, p. 154).

O motivo de ressaltar essa preocupação para a elaboração de propostas didáticas está na possibilidade de seleção e simplificação adequada de um episódio histórico, de modo que possa representá-lo sem excluir informações importantes e, sem deixar de projetar as atividades para o desenvolvimento das habilidades cognitivas relacionadas ao pensamento crítico, como foi possível verificar em Matthews (1995) e Tenreiro-Vieira Vieira (2000).

Essa é uma tarefa complexa, que exige realizar atividades, na dinâmica da sala de aula, para estimular o entendimento dos problemas relacionados ao desenvolvimento do conhecimento científico (CARVALHO; GIL-PÉREZ, 2011; MATTHEWS, 1995). Assim como destacado de Peduzzi ao discutir que "é sem dúvida, a pesquisa, que em condições de sala de aula e com materiais históricos apropriados, de boa qualidade, que vai referendar ou refutar afirmações [...]" (2005, p. 157).

Por isso, para favorecer a discussão do processo de construção do conhecimento científico abordado em um episódio histórico é necessário compreender que esse processo envolve habilidades necessárias para serem desenvolvidas pelos estudantes no ensino de Ciências, a exemplo do destaque de Matthews: "distinguir entre asserções e argumentos pautados em dados e prova científica e os que não o são" (1995, p. 4), apresentadas pelo Conselho Nacional do Currículo (representado em inglês pela sigla NCC), no ano de 1988 ao tratar sobre a reforma e inovação curricular. Nesse sentido, o recorte a seguir destaca que:

Face ao progresso atual, a grande maioria dos conhecimentos válidos hoje estarão obsoletos num curto intervalo de tempo. Além disso, dada a multiplicação galopante do conhecimento disponível no mundo, é difícil, se não impossível, prever qual a informação de que os indivíduos irão necessitar no futuro (TENREIRO-VIEIRA VIEIRA 2000, p.15)

Dessa maneira, a educação científica passa a exigir práticas de ensino nas quais os estudantes participem de atividades que exijam raciocínio lógico para solucionar o problema que está em causa, que tomem partido em questões que envolvem aspectos do conhecimento científico, que saibam detectar inconsistências na argumentação apresentada ou até em uma tomada de decisão emitida, quando as evidências não forem suficientes para elaborar um caminho e sustentar uma conclusão (CACHAPUZ et al., 2005; MATTHEWS, 1994; TENREIRO-VIEIRA; VIEIRA, 2000, 2005).

Para tanto, este estudo finaliza suas explanações com uma reflexão sobre as suas implicações no ensino de Ciências, pois, as correlações explícitas realizadas, que representam as dimensões teóricas de conhecimento para a tomada de decisão, podem ser inseridas no ensino de Ciências se forem consideradas como uma base para a proposição de materiais didáticos (CARDOSO, 2021).

As atividades de aprendizagem, a serem projetadas por meio dessas correlações, podem viabilizar a análise de informações de textos utilizados para problematizar um episódio histórico da construção do conhecimento científico, ou, as atividades de análise de informações podem culminar na distinção de afirmações e argumentos tidos como científicos dos que não são, em atividades de debates do episódio histórico ou de pesquisa em fontes de informação, em um processo de busca, seleção e julgamento de informações atribuídas como confiáveis, mediante 
critérios e procedimentos adequados (CARDOSO, 2021; HALPERN, 1999; MATTHEWS, 1995; TENREIRO-VIEIRA; VIEIRA, 2000, 2005).

Outra possibilidade é a projeção de atividades experimentais que procuram distanciarse da tendência de procedimentos de receita de bolo, e objetivam ter o potencial de investigação, elas podem concentrar as três dimensões de problematização, análise e distinção do conhecimento científico para a tomada de decisão, assim como podem ser uma base em abordagens de estudos de caso, de modo a salientar nos estudantes o desenvolvimento de habilidades cognitivas do Pensamento Crítico, relacionadas à formulação de hipóteses e busca por evidências que sustentem suas respostas para os problemas propostos (MATTHEWS, 1995; TENREIRO-VIEIRA; VIEIRA, 2000, 2005, 2006).

Um exemplo concreto dessas projeções pode ser evidenciado no estudo realizado por Cardoso (2021), na qual essas correlações passaram por um processo de validação com professores-pesquisadores do ensino de Ciências, ao avaliarem uma Sequência de EnsinoAprendizagem que aborda a controvérsia científica entre Luigi Galvani e Alessandro Volta (século XVIII), de modo que foi possível evidenciar a importância dessas correlações no estabelecimento de uma proposta com potencial para a promoção e mobilização de capacidades de Pensamento Crítico em estudantes da Educação Básica, conforme a tipologia apresentada por Tenreiro-Vieira e Vieira (2005).

\section{Considerações finais}

Em se tratando das correlações implícitas, apesar de não serem alvo de inserção direta em atividades de aprendizagem, são considerações importantes para justificar a complexidade da formação científica para o desenvolvimento da cidadania, que é tão promulgada por pesquisas no ensino de Ciências. Essas correlações implícitas possibilitam compreender que as dimensões de História da Ciência e do pensamento crítico também compartilham objetivos para a educação científica de qualidade, além de permitir direcionar qual o ponto de intersecção dentre as suas aproximações teóricas que exige uma formação para a cidadania, uma vez que é tão complexo discuti-la em função dos diversos referenciais e objetivos plurais que seu termo comporta.

No caso das dimensões explícitas, as suas correlações tornam possível a projeção de atividades que tenham o conhecimento como pressuposto a ser utilizado na tomada de decisão, o que pode contribuir para mobilizar os recursos intelectuais relacionados ao conhecimento científico e às habilidades cognitivas de forma interrelacionada. Portanto, a abordagem teórica apresentada pela convergência de aspectos comuns entre os objetivos da História da Ciência e do pensamento crítico se revela como um possível contributo ao propor um modelo teórico que objetiva a projeção dessas dimensões em propostas didáticas para o ensino de ciências. Enquanto proposição de modelo teórico, isso não impede ou limita a projeção de práticas de ensino, mas este estudo o destaca como uma tentativa de orientar a projeção dessas complexas dimensões de forma integrada no ensino de Ciências, e pode ser um incentivo para as novas propostas teóricas e/ou práticas que podem surgir.

\section{Agradecimentos}

O presente trabalho foi realizado com apoio da Coordenação de Aperfeiçoamento de Pessoal de Nível Superior - Brasil (CAPES) - Código de Financiamento 001.

\section{Referências}


ALLCHIN, D. Pseudohistory and Pseudoscience. Science \& Education, v. 13, p. 179-195, 2004.

BARDIN, L. Análise de Conteúdo. Tradução: Luís Antero Reto e Augusto Pinheiro. São Paulo: Edições 70, 2011.

CACHAPUZ, A. et al. Importância da educação científica na sociedade atual. In:

CACHAPUZ, A., et al. A necessária renovação do ensino das Ciências. São Paulo: Cortez Editora, 2005. p. 19-34.

CARDOSO, S. C. Capacidades de Pensamento Crítico a partir de uma Abordagem Contextual para o ensino de Eletroquímica. 2021. 190 f. Dissertação (Mestrado em Ensino de Ciências e Matemática) - Universidade Federal Sergipe, São Cristóvão, 2021.

CARVALHO, A. M. P.; GIL-PÉREZ, D. Formação de professores de Ciências: tendências e inovações. 10a. ed. São Paulo: Cortez, v. 28, 2011.

DUARTE, M. C. A História da Ciência na prática de professores portugueses: implicações para a formação de professores de ciências. Ciência e Educação, v. 10, n. 3, p. 317-331, 2004.

EL-HANI, C. N. Notas sobre o ensino de História e Filosofia da Ciência na educação científica de nível superior. In: SILVA, C. C. Estudos de História e Filosofia das Ciências: subsídios para aplicação no ensino. 1. ed. São Paulo: Livraria da Física, 2006. p. 3-21.

ENNIS, R. H. A logical basis for measuring critical thinking skills. Educational Leadership, v. 43, n. 2, p. 44-48, 1985.

FORATO, T. C. M.; PIETROCOLA, M.; MARTINS, R. A. Historiografia e natureza da ciência na sala de aula. Caderno Brasileiro de Ensino de Física, v. 28, n. 1, p. 27-59, 2011.

FORATO, T. C. M.; PIETROCOLA, M.; MARTINS, R. A. Enfrentando obstáculos na transposição didática da História da Ciência para a sala de aula. In: PEDUZZI, L. O. Q.; MARTINS, A. F. P.; FERREIRA, J. M. H. Temas de História e Filosofia da Ciência no ensino. Natal: EDUFRN, 2012. p. 123-154.

FREIRE JUNIOR, O. A relevância da Filosofia e História das Ciências para formação dos professores de ciências. In: SILVA FILHO, W. J. Epistemologia e ensino de ciências. Salvador: Arcádia, 2002. p. 13-30.

FREIRE, L. I. F. Pensamento Crítico, enfoque CTS e o ensino de Química. 2007. 175 f. Dissertação (Mestrado em Educação Científica e Tecnológica) - Universidade Federal de Santa Catarina, Florianópolis. 2007.

GAGLIARDI, R. Cómo utilizar la Historia de las Ciencias en la enseñanza de las Ciencias. Enseñanza de las Ciencias, v. 6, n. 3, p. 291-296, 1988. 
GURGEL, I. Reflexões político-curriculares sobre a importância da História das Ciências no contexto da crise da modernidade. Caderno Brasileiro de Ensino de Física, v. 37, n. 2, p. 333-350, 2020.

HALPERN, D. F. Teaching for critical thinking: helping college students develop the skills and dispositions of a critical thinker. In: [S. n.] New directions for teaching and learning. [S. 1.]: Jossey-Bass Publishers, 1999. p. 69-74.

HIDALGO, M. R.; LORENCINI JUNIOR, Á. Reflexões sobre a inserção da História e Filosofia da Ciência no ensino de Ciências. História da Ciência e Ensino: construindo interfaces, v. 14, p. 19-38, 2016.

KIPNIS, N. Scientific controversies in teaching science: the case of Volta. In: BEVILACQUA, F.; GIANNETTO, E.; MATTHEWS, M. R. Science Educational and Culture. Dordrecht/ Boston/ London: Kluwer Academic Publishers, 2001. p. 255-271.

LIPMAN, M. Critical Thinking - What can it be? Educational Ledearship, v. 46, n. 1, p. 3843, 1988.

MARTINS, A. F. P. História e Filosofia da Ciência no ensino: há muitas pedras nesse caminho. Caderno Brasileiro de Ensino de Física, v. 24, n. 1, p. 112-131, 2007.

MARTINS, R. A. Sobre o papel da História da Ciência no ensino. Boletim da Sociedade Brasileira de História da Ciência, v. 9, p. 3-5, 1990.

MARTINS, R. A. Introdução: a História das Ciências e seus usos na educação. In: SILVA, C. C. Estudos de História e Filosofia das Ciências: subsídios para aplicação no ensino. São Paulo: Livraria da Física, 2006. p. 17-30.

MATTHEWS, M. R. Science teaching: the role of History and Philosophy of Science. New York: Routlegde, 1994.

MATTHEWS, M. R. História, Filosofia e ensino de Ciências: a tendência atual de reaproximação. Caderno Catarinense de Ensino de Física , v. 12, n. 3, p. 164-214, 1995.

MATTHEWS, M. R. O tempo e o ensino de ciência: como o ensino de História e Filosofia do movimento pendulr pode contribuir para a alfabetização científica. In: SILVA FILHO, W. J. Epistemologia e ensino de Ciências. Salvador: Arcádia, 2002. p. 31-48.

MOURA, C. B.; GUERRA, A. História Cultural da Ciência: um caminho possível para a discussão de práticas científicas no ensino de Ciências? Revista Brasileira de Pesquisa em Educação em Ciências, v. 16, n. 3, p. 725-748, 2016.

OLIVEIRA, R. A.; SILVA, A. P. B. A História da Ciência no ensino: diferentes enfoques e suas implicações na compreensão da ciência. In: VIII Encontro Nacional de Pesquisa em Educação em Ciências e I Congresso Iberoamericano de Investigación en Enseñanza de las Ciencias. Campinas. Anais [...]. Campinas, 2011. Disponível em:

<http://abrapecnet.org.br/atas_enpec/viiienpec/lista_area_3.htm.> Acesso em: 5 abr. 2020. 
PEDUZZI, L. O. Q. Sobre a utilização didática da História da Ciência. In: PIETROCOLA, M. Ensino de Física: conteúdo, metodologia e epistemologia em uma concepção integradora. $2^{\mathrm{a}}$. ed. Florianópolis: Editora da UFSC, 2005. p. 151-170.

SEQUEIRA, M.; LEITE, L. A História da Ciência no ensino - aprendizagem das ciências. Revista Portuguesa de Educação, Braga, v. 1, n. 2, p. 29-40, 1988.

SIEGEL, H. The racionality of Science, critical thinking and science educaction. Synthese, v. 80, n. 1, p. 9-41, 1989.

SILVA, E. L. Contribuições da eleaboração de Sequências de Ensino Aprendizagem tratando das tendências de Interdisciplinaridade, Cotidiano e História da Ciência no âmbito da formação de professores da Universidade Federal de Sergipe. 2014. 184 f. Tese (Doutorado em Ensino, Filosofia e História das Ciências) - Universidade Federal da Bahia / Universidade Estadual de Feira de Santana, Salvador. 2014.

TENREIRO-VIEIRA, C. Produção e avaliação de atividades de aprendizagem de ciências para promover o Pensamento Crítico dos alunos. Revista Iberoamericana de Educación, p. $1-17,2004$.

TENREIRO-VIEIRA, C.; VIEIRA, R. M. Promover o Pensamento Crítico dos alunos: propostas concretas para a sala de aula. Porto: Porto Editora, 2000.

TENREIRO-VIEIRA, C.; VIEIRA, R. M. Estratégias de ensino/aprendizagem. Lisboa: Instituto Piaget, 2005.

TENREIRO-VIEIRA, C.; VIEIRA, R. M. Produção e validação de actividades de laboratório promotoras de Pensamento Crítico dos alunos. Revista Eureka sobre Enseñanza y Divulgación de las Ciencias, v. 3, n. 3, p. 4452-466, 2006.

TENREIRO-VIEIRA, C.; VIEIRA, R. M. Práticas didático-pedagógicas de Ciências: estratégias de ensino-aprendizagem promotoras do Pensamento Crítico. Saber e Educar, p. 34-41, 2015.

TENREIRO-VIEIRA, C.; VIEIRA, R. M. Promover o pensamento crítico em ciências na escolaridade básica: propostas e desafios. Revista Latinoamericana de Estudios Educativos, v. 15, n. 1, p. 36-49, 2019.

TENREIRO-VIEIRA, C.; VIEIRA, R. M. Promover o Pensamento Crítico em contextos CTS: desenvolvimento de propostas didáticas para o Ensino Básico. Indagatio Didactica, v. 12, n. 4, p. 471-484, 2020.

TENREIRO-VIEIRA, C.; VIEIRA, R. M. Promover o Pensamento Crítico e Criativo no ensino das Ciências: propostas didáticas e seus contributos em alunos portugueses.

Investigações em Ensino de Ciências, v. 26, n. 1, p. 70-84, 2021. 